\title{
DIZZYNET 2019: approaching the future of vestibular research
}

\author{
Andreas Zwergal $^{1,2} \cdot$ Eva Grill $^{2,3} \cdot$ Christophe Lopez ${ }^{4} \cdot$ Marianne Dieterich $^{1,2}$
}

Published online: 28 August 2019

(c) Springer-Verlag GmbH Germany, part of Springer Nature 2019

Keywords European dizzynet $\cdot$ Vertigo $\cdot$ Dizziness $\cdot$ Balance disorders $\cdot$ Vestibular

Vestibular research is undergoing fundamental changes as new technological developments arise. The most rapidly emerging fields include the following: (1) use of digital applications and mobile devices to track the clinical course of patients with recurrent vestibular disorders [1]. (2) Selfvideo recording of eye and body movements during vestibular attacks [2]. (3) Refinement of imaging methods to depict pathological changes in the inner ear and central vestibular networks [3, 4]. (4) Increasing practical application of innovative therapeutic strategies (e.g., vestibular implants, stochastic galvanic vestibular stimulation, vestibulo-ocular reflex adaptation) [5]. (5) Improved pathophysiological understanding of vestibular disorders by analysing data from genomics or proteomics [6]. (6) Application of modern data mining techniques to optimize diagnostic decisionmaking [7]. These developments not only have enormous potential for future vestibular research and new therapies, but also require consequent transnational and multiprofessional cooperation. DIZZYNET is dedicated to intensify and strengthen the interdisciplinary collaboration to face these future challenges in vestibular research.

This manuscript is part of a supplement sponsored by the German Federal Ministry of Education and Research within the funding initiative for integrated research and treatment centers.

Andreas Zwergal

andreas.zwergal@med.uni-muenchen.de

1 Department of Neurology, Ludwig-Maximilians-University, Marchioninistrasse 15, 81377 Munich, Germany

2 German Center for Vertigo and Balance Disorders, DSGZ, Ludwig-Maximilians-University, Munich, Germany

3 Institute for Medical Information Processing, Biometry, and Epidemiology (IBE), Ludwig-Maximilians-University, Munich, Germany

4 Laboratory of Sensory and Cognitive Neuroscience, CNRS, Aix Marseille Université, Marseille, France
At the fifth DIZZYNET meeting, which took place in Starnberg near Munich in October 2018, 80 experts from 20 European countries and experts from the US and Australia (David S. Zee, Michael G. Halmagyi), discussed innovations in vestibular research and decided on collaborate actions (e.g., an app-based patient diary, data banking of multimodal data for vestibular disorders). Selected contributions from this meeting covering all areas of vestibular research are presented in the current DIZZYNET issue of the Journal of Neurology. A number of articles focus on vestibular rehabilitation and therapy. Klaus Jahn summarizes the specific needs of the vestibular rehabilitation community across Europe. Dara Meldrum gives a comprehensive overview of the current developments in gaze stabilization exercises. Nils Guinand presents original data suggesting that the vestibular implant effectively modulates vestibulospinal projections involved in control of posture. Christian Chabbert describes new insights into mechanisms of peripheral vestibular regeneration as a potential target for future treatments. In a Xenopus experiment, Hans Straka suggests a mode of action of 4-aminopyridine on irregular vestibular afferents. Endolymphatic hydrops (ELH) is addressed by Roberto Teggi from the molecular viewpoint of ion transporters, and by Valerie Kirsch from an inner ear imaging perspective. A novel segmentation approach of the inner ear fluid space is described that is based on a probabilistic atlas and might give rise to an improved automated analysis of ELH in the future. Andreas Schepermann and Max Wuehr report novel experimental models for Mal de Debarquement Syndrome and fear of heights using motion platforms and virtual reality environments. Several articles discuss new aspects of higher vestibular function. In a large cohort study, Marianne Dieterich and colleagues illustrate that residual vestibular function is relevant to vertigo-related anxiety. Christophe Lopez presents an elegant study in patients with unilateral vestibular failure, who show impaired embodied spatial cognition. Another study describes a novel approach 
to assess spatial orientation and memory using a cell phonebased bedside approach. Finally, an analysis of more than 5 million data sets from an insurance company shows that the rate of car accidents is not increased in patients with acute unilateral vestibulopathy and Menière's disease. These data should be taken into consideration for future amendments of driving license regulations in European countries.

The above-mentioned articles demonstrate the broad and innovative spectrum of topics from basic research to clinical application and therapy represented by the DIZZYNET members and the specific advantages of the international DIZZYNET as a collaborative platform for launching future vertigo and balance research projects.

Acknowledgements The DIZZYNET initiative was supported by the German Federal Ministry of Education and Health (BMBF) within the context of the foundation of the German Center for Vertigo and Balance Disorders (DSGZ) (Grant no. 01 EO 0901). The authors thank Katie Göttlinger for critically reading and copy-editing the manuscript.

\section{Compliance with ethical standards}

Conflicts of interest The authors declare no competing financial interests.

\section{References}

1. Martin E, Leue C, Delespaul P et al (2019) An app-based diary for vestibular disorders: the dizzyquest. J Psychosom Res 121:111-112

2. Young AS, Lechner C, Bradshaw AP et al (2019) Capturing acute vertigo: a vestibular event monitor. Neurology 92(24):e2743-e2753

3. Lopez-Escamez JA, Attyé A (2019) Systematic review of magnetic resonance imaging for diagnosis of Meniere disease. J Vestib Res 29(2-3):121-129

4. Popp P, Zu Eulenburg P, Stephan T et al (2018) Cortical alterations in phobic postural vertigo-a multimodal imaging approach. Ann Clin Transl Neurol 5(6):717-729

5. Schubert MC, Migliaccio AA (2019) New advances regarding adaptation of the vestibulo-ocular reflex. J Neurophysiol 122(2):644-658

6. Gallego-Martinez A, Espinosa-Sanchez JM, Lopez-Escamez JA (2018) Genetic contribution to vestibular diseases. J Neurol 265(Suppl 1):29-34

7. Grill E, Groezinger M, Feil K et al (2016) Developing and implementing diagnostic prediction models for vestibular diseases in primary care. Stud Health Technol Inform 228:735-739 